\title{
Die gastroösophageale Refluxkrankheit in der Schwangerschaft
}

\author{
Sara Fill Malfertheiner, Birgit Seelbach-Göbel
}

\section{Übersicht}

Einleitung

GERD im Allgemeinen

GERD in der Schwangerschaft

GERD in der Laktationsphase

\section{Einleitung}

Die gastroösophageale Refluxkrankheit (GERD) ist die häufigste gastrointestinale Erkrankung in den Industrieländern und der westlichen Welt [1]. GERD wird im Jahre 2015 mit einer Prävalenz von bis zu 15\% beschrieben und zeigt eine zunehmende Inzidenz [2].

Eine gastroösophageale Refluxkrankheit entwickelt sich, wenn der Reflux von Mageninhalt störende Symptome und/oder Komplikationen verursacht. Zu den Komplikationen zählt auch eine signifikante Störung des gesundheitsbezogenen Wohlbefindens (Lebensqualität) [3]. Refluxbeschwerden entstehen, wenn mit Magensäure vermischter Speisebrei aus dem Magen in die Speiseröhre zurückfließt und die Speiseröhrenschleimhaut reizt. Durch diese Reizung entstehen brennende Schmerzen hinter dem Brustbein, sog. Sodbrennen. Sodbrennen ist das Hauptsymptom von GERD.

Ursache für GERD ist eine pathologische Funktion des unteren Ösophagussphinkters.

Auch in der Schwangerschaft ist GERD eine häufige Erkrankung. GERD tritt bei mehr als 50\% der Frauen im Verlauf einer Schwangerschaft auf [4]. GERD wird oft als schwangerschaftsimmanente Erscheinung interpretiert. Es ist aber wichtig zu wissen, dass GERD die Lebensqualität der Schwangeren erheblich beeinträch- tigen kann [5]. Wie auch außerhalb der Schwangerschaft, ist eine gestörte Verschlussfunktion des unteren Ösophagussphinkters die Hauptursache für GERD. In der Schwangerschaft wird dieser Verschlussmechanismus durch schwangerschaftsbedingte Veränderungen der Sexualhormone, insbesondere durch das Progesteron hervorgerufen [6]. Das häufigste Symptom der GERD in der Schwangerschaft ist das Sodbrennen. Komplikationen von GERD wie beispielsweise eine erosive Ösophagitis sind während der Schwangerschaft selten beschrieben.

Mehr als $50 \%$ aller schwangeren Frauen leiden im Verlauf der Schwangerschaft unter GERD.

Die Therapie von GERD in der Schwangerschaft folgt einem empfohlenen „Step-up“-Algorithmus, beginnend mit spezifischen Lebensstil-Änderungen und Veränderungen des Essverhaltens [2,6]. Eine übermäßige Gewichtszunahme in der Schwangerschaft sollte vermieden werden. Ist eine medikamentöse Therapie erforderlich, so sind Antazida die Medikamente der ersten Wahl. Wenn die Symptome persistieren, stehen Histamin-2-Rezeptorantagonisten sowie Protonenpumpen-Inhibitoren (PPI) zur Verfügung. PPI sind laut der neuesten S2k-Leitlinie der Deutschen Gesellschaft für Gastroenterologie, Verdauungs- und Stoffwechselkrankheiten (DGVS) bei persistierenden GERD-Symptomen empfohlen. Die GERD-Symptomatik bessert sich nach der Entbindung meist rasch, über die Spätfolgen bzw. den Einfluss auf nachfolgende 
Schwangerschaften ist wenig bekannt. Die Literatur zeigt, dass GERD in der Schwangerschaft oft nicht oder inadäquat behandelt wird [5].

Aufgrund der hohen Prävalenz spielt GERD auch hinsichtlich des gesundheitsökonomischen Aspektes eine große Rolle. Der Artikel stellt eine Aktualisierung und Zusammenführung des 2007 veröffentlichen Reviews (ZGN) zum Thema: „Management der Gastroösophagealen Refluxkrankheit in der Schwangerschaft" [4] und der 2014 neu veröffentlichten Leitlinie zur „Gastroösophagealen Refluxkrankheit“, unter Federführung der DGVS (AWMF-Register-Nr. 021-013) dar [2].

\section{GERD im Allgemeinen}

GERD ist eine der häufigsten gastrointestinalen Erkrankungen in den Industriestaaten. Sie ist inzwischen die häufigste säurebedingte Erkrankung des oberen Gastrointestinaltrakts und wird mit einer Prävalenz von mindestens $15 \%$ beschrieben [2]. Sodbrennen wird als das häufigste Symptom der GERD beschrieben, von dem mehrere Millionen Menschen in Deutschland betroffen sind.

Die aktuell gültige Montreal-Klassifikation soll auf die Aspekte von Nomenklatur, Diagnostik und Therapie der gastroösophagealen Refluxkrankheit (GERD) angewandt werden (Abb. 1) [3]. Eine Refluxkrankheit kann zu entzündlichen Schleimhautreaktionen, sog. Erosionen, führen. Sind solche organischen Manifestationen nachweisbar, spricht man von dem Krankheitsbild der erosiven Refluxkrankheit, ERD. Von einer ERD sind jedoch weniger als $50 \%$ aller Patienten mit typischen GERD-Symptomen betroffen [2]. Eine GERD ohne endoskopisch nachweisbare Läsionen (Erosionen, Striktur, Barrett-Ösophagus) wird unter dem Begriff nichterosive gastroösophageale Refluxkrankheit (NERD) definiert [2]. Die Lebensqualität von GERDPatienten ist durch die unterschiedlichsten Symptome stark beeinträchtigt, zeigt aber keine Korrelation mit dem Schweregrad einer endoskopischen Ösophagitis [2].

Die Lebensqualität der Patienten mit GERD ist durch mannigfaltige Symptome stark beeinträchtigt.

\section{Definition}

Zur Diagnosestellung der GERD orientiert man sich an der aktuell gültigen Montreal-Klassifikation (Abb. 1): Eine GERD wird hervorgerufen, wenn der Reflux von Mageninhalt störende Symptome und/oder Komplikationen verursacht [3]. In den Montreal-Kriterien werden auch asymptomatische Patienten erfasst, die jedoch eine GERD-Komplikation (z. B. Barrett-Ösophagus) aufweisen. Zudem werden Patienten mit GERD diagnostiziert, die lediglich Symptome aufweisen ohne nachweisbare Komplikationen oder organische Veränderungen. Auch alle endoskopisch diagnostizierten Manifestationen (Refluxnachweis mit pH-Metrie, Impedanz), auch wenn keine Symptome bestehen, fallen

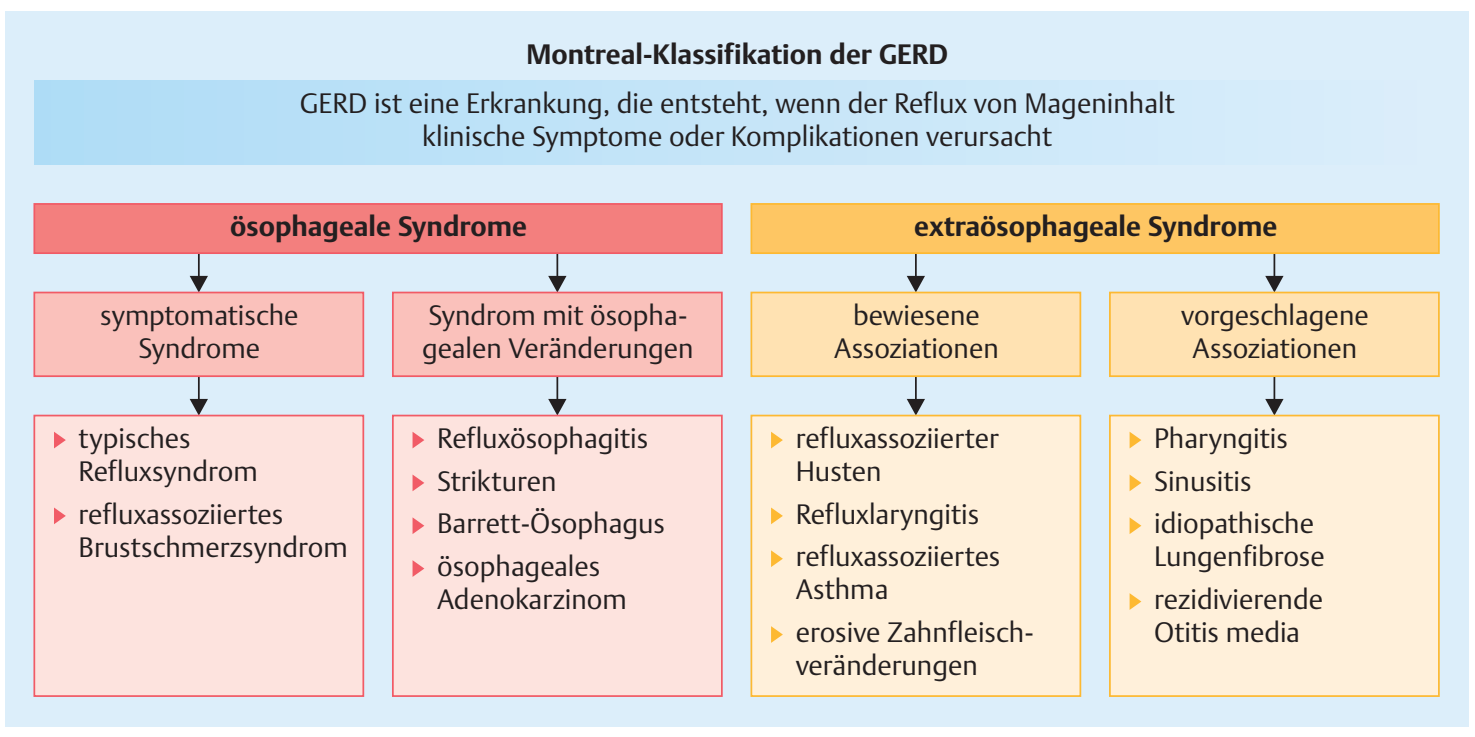

Abb. 1 Die Montreal-Klassifikation (nach [3]). 
unter das Krankheitsbild der GERD. In einer skandinavischen Studie, der sog. Kalixandra-Studie, konnte bei $36,5 \%$ von symptomfreien Personen eine Refluxösophagitis nachgewiesen werden, sodass daher auch symptomlose Ösophagitiden als GERD definiert werden [7]. Andererseits klassifiziert die Studie GERD auch beim alleinigen Nachweis von Refluxfolgen (Endoskopie, Histologie, Elektronenmikroskopie) und deren Symptomatik.

\section{Tipp für die Praxis}

Eine GERD wird als wahrscheinlich angesehen, wenn typische Refluxsymptome (Sodbrennen, Regurgitation) mindestens $1 \times /$ Woche bis $2 \times /$ Woche auftreten und mit einer Beeinträchtigung der Lebensqualität einhergehen.

Führt Sodbrennen die klinische Symptomatik an, so liegt mit hoher Wahrscheinlichkeit eine Refluxerkrankung vor.

Die Montreal-Klassifikation ist durch deutsche und italienische Expertengruppen validiert und findet Anwendung in amerikanischen und europäischen Leitlinien [2].

\section{Klinische Manifestation}

Typische und häufige Symptome von GERD sind Sodbrennen und saures Aufstoßen, wobei Sodbrennen als Hauptsymptom der GERD definiert wird [2]. Unter Sodbrennen versteht man ein hinter dem Brustbein lokalisiertes Gefühl von Brennen und Hitze, das vom mittleren Oberbauch bis in die Halsgrube aufsteigt. Ösophageale sowie extraösophageale Symptome von GERD sind oft:

- das Aufstoßen von Luft und saurem Magensaft

- unangenehme Aufwärtsbewegungen von Mageninhalten

- Druck- und Völlegefühl im Oberbauch

- Schmerzen hinter dem Brustbein

- Kloßgefühl im Hals

- Symptome der Atemwege wie Heiserkeit, Husten und Dyspnoe

- eine verstärkte Speichelsekretion [2]

Die Symptome erlauben keinen definitiven Rückschluss auf pathologische Schleimhautveränderungen in der unteren Speiseröhre [2].

\section{Cave}

Zu den etablierten extraösophagealen Symptomen zählen Laryngitis, chronischer Husten, Asthma und Zahnerosionen. In Diskussion ist auch eine Assoziation zwischen GERD und Pharyngitis, Sinusitis, idiopathischer Lungenfibrose und rezidivierender Otitis media. Eine enge Korrelation von GERD zu Atemwegserkrankungen, Asthma und Schnarchen ist ebenso beschrieben wie zu nächtlicher Insomnie, Benzodiazepin-Abusus, Müdigkeit am Tage und Bluthochdruck.

Unter GERD werden zudem weitere unterschiedliche Krankheitsbilder zusammengefasst [2]:

- extraösophageale Manifestationen

- Komplikationen der GERD

- erosive Refluxösophagitis verschiedener Schweregrade (ERD)

- nichterosive Refluxkrankheit (NERD)

- hypersensitiver Ösophagus

- funktionelle Refluxbeschwerden

- Barrett-Ösophagus

\section{Extraösophageale Manifestationen und Komplikationen der GERD}

Komplikationen der GERD können sich in der Speiseröhre sowie extraösophageal „atypisch“ manifestieren. Zu den etablierten extraösophagelen Symptomen zählen Laryngitis, chronischer Husten, Asthma und Zahnerosionen. Eine Assoziation zwischen GERD und idiopathischer Lungenfibrose, rezidivierender Otitis media, Pharyngitis und Sinusitis wird vermutet [2]. Eine enge Korrelation von GERD zu Atemwegserkrankungen, Asthma, nächtlicher Insomnie, BenzodiazepinAbusus, Müdigkeit am Tage, Schnarchen sowie Bluthochdruck ist bereits beschrieben [8]. GERD und obstruktive Schlafapnoe sowie Helicobacter pylori zeigen keine Korrelation [9]. GERD ist zudem die häufigste ösophageale Ursache des Noncardiac Chest Pain, welches auch als atypische Manifestationsform der GERD aufgelistet ist [10]. Komplikationen am Ösophagus können beispielsweise Strikturen, Ösophagitiden oder ein Barrett-Ösophagus sein.

GERD ist die häufigste Ursache des Noncardiac Chest Pain. 
- Erosive Refluxkrankheit (ERD)

GERD mit endoskopisch nachweisbaren Läsionen wird als erosive gastroösophageale Refluxkrankheit definiert. Zu eventuellen organisch nachweisbaren Läsionen zählen Strikturen, Erosionen sowie ein BarrettÖsophagus. Weniger als 50\% aller Patienten mit typischen GERD-Symptomen haben endoskopisch erkennbare Schleimhautläsionen [2]. Patienten mit einer Hiatushernie oder starkem Alkohol- und Nikotinkonsum, Adipositas, männlichem Geschlecht und Helicobacterpylori-Negativität haben ein erhöhtes Risiko für die Entwicklung einer ERD [2].

\section{nichterosive Refluxerkrankung (NERD)}

GERD ohne endoskopisch nachweisbare organische Läsionen wird als nichterosive gastroösophageale Refluxkrankheit definiert [2]. Mehr als die Hälfte der Patienten mit typischen GERD-Symptomen sind von einer NERD betroffen [2]. Sie liegt vor, wenn die Beschwerden die Lebensqualität beeinträchtigen. NERD kann die Lebensqualität ähnlich stark beeinträchtigen wie ERD. Patienten mit einer NERD haben häufiger einen niedrigen BMI und den Nachweis einer Helicobacter-pylori-Infektion. Jüngere Frauen sind häufiger betroffen $[7,9,11]$.

\section{- Hypersensitiver Ösophagus}

Patienten, die keinen Nachweis eines pathologisch veränderten Reflux aufweisen, bei denen jedoch der in der Norm liegende Reflux typische Symptome ohne endoskopisch nachweisbare Läsionen auslöst, leiden an einem hypersensitiven Ösophagus [2]. Bei dieser Symptomatik ist eine säuresuppressive Therapie erfolgversprechend.

\section{- Funktionelle Refluxbeschwerden}

Patienten mit funktionellen Refluxbeschwerden klagen zwar über Sodbrennen, es liegt aber weder ein nachweisbarer pathologischer Reflux vor noch besteht eine zeitliche Assoziation der Schmerzangaben mit physiologischen Refluxereignissen. Eine erhöhte afferente ösophageale Sensitivität wird vermutet [2]. Bei dieser Symptomatik ist eine säuresuppressive Therapie ineffektiv. Unter dieser Symptomatik leiden häufiger jüngere Frauen mit psychologischer Komorbidität [10].

\section{narrett-Ösophagus}

Der Begriff Barrett-Ösophagus beschreibt einen Zustand, bei dem das normale Plattenepithel des Ösophagus am gastroösophagealen Übergang durch abnormales, spezialisiertes Zylinderepithel ersetzt wird. Diese Umwandlung von ösophagealem Plattenepithel zu Zylinderepithel nennt man intestinale Metaplasie. Die Diagnose wird bei endoskopisch-makroskopischem Verdacht histologisch gestellt. Als Standard für die endoskopische Diagnostik bei Patienten mit BarrettÖsophagus gilt heutzutage die hochauflösende Videoendoskopie.

Ein Barrett-Ösophagus entwickelt sich bei ca. 10\% der Menschen mit chronischem gastroösophagealem Reflux.

Weder die Frequenz noch die Schwere der Refluxsymptome sind prädiktive Faktoren für die Entstehung oder die Ausdehnung einer solchen Metaplasie. Gleichzeitig stellt der Barrett-Ösophagus eine präkanzeröse Veränderung dar, aus der sich im Verlauf eine Neoplasie entwickeln kann. Das Risiko der Progression eines nicht neoplastischen Barrett-Ösophagus hin zur hochgradigen intraepithelialen Neoplasie oder zum Adenokarzinom ist äußerst niedrig und wird in neueren Studien mit 0,12-0,33 pro Jahr angegeben $[2,11]$.

\section{Therapie}

Vor einem Therapiebeginn bei einer GERD ist eine genaue Diagnostik notwendig. Dazu zählt eine genaue Anamnese hinsichtlich der typischen Refluxbeschwerden (z. B. Sodbrennen, Säureregurgitation) sowie möglicher refluxassoziierter Symptome (z. B. epigastrische Schmerzen, thorakale Schmerzen, Dysphagie, Odynophagie, Brennen im Rachen, Räuspern). Zudem müssen mögliche Beschwerden mit Hinweis auf funktionelle Erkrankungen (z. B. Symptome des Reizmagens und Reizdarms) eruiert werden.

Medikamente können GERD verursachen, eine vorbestehende GERD-Symptomatik durch die Beeinflussung der Motilität verstärken, zu einer Relaxation des unteren Ösophagussphinkters führen oder die ösophageale Clearance stören.

Eine ausführliche Medikamentenanamnese ist zur Therapie erforderlich. 
Medikamente, die auf das Krankheitsbild GERD einen Einfluss haben, sind in der aktuellen Leitlinie genannt:

- Kalziumantagonisten

- Nitropräparate

- Theophylline und Anticholinergika

- beta-adrenerge Agonisten

- Benzodiazepine

- pfefferminzhaltige Präparate

- Östrogenpräparate zur postmenopausalen Hormontherapie

Aminophylline können zudem zu einer Verstärkung eines refluxbedingten Asthmas führen. Gestagene und Antikonzeptiva haben dagegen keinen gesicherten $\mathrm{Zu}$ sammenhang mit GERD. Wiederum andere Medikamente können eine motilitätsunabhängige, toxische Ösophagitis direkt lokal (ASS, NSAR, Bisphosphonate, Doxycylin, Eisensulfat, Ascorbinsäure) oder systemisch auslösen (Zytostatika) [2].

Nach einer ausführlichen Anamnese ist das wichtigste Therapieziel für GERD, die Symptome zu lindern bzw. zu beseitigen.

Jede Therapie sollte allgemeine Ratschläge zur Lebensstil-Änderung enthalten.

\section{Tipp für die Praxis}

Vorschläge zur Lebensstil-Änderung sind:

- Aufforderung zu gesünderem Leben

- unverträgliche sowie magenreizende Nahrungsmittel meiden

- das Rauchen aufgeben

- bei Übergewicht abnehmen

- Stress entgegenwirken

- für besseren Schlaf evtl. das Kopfteil des Bettes leicht erhöhen

PPI-Therapie. Weist ein Patient typische Refluxsymptome einer GERD ohne gefährliche Alarmsymptome auf, kann bei Erwachsenen eine empirische Protonenpumpenhemmer-Therapie ohne weitere Diagnostik erfolgen. Wichtig ist zu eruieren, ob aus der Krankheitsdauer kein relevantes Risiko eines Barrett-Ösophagus resultiert [2].

Als Alarmsymptome sind in der aktuellen Leitlinie die folgenden beschrieben:

- Dysphagie und Odynophagie

- unfreiwilliger Gewichtsverlust (>5\%)

- Anämie, insbesondere bei klinischen Hinweisen auf GI-Blutverluste
- klinische/apparative Hinweise auf eine ösophageale/ epigastrische Raumforderung, Striktur oder ein Ulkus [2]

Weiterführende Diagnostik. Sind die Symptome einer Refluxkrankheit jedoch nicht eindeutig, muss eine weitere Diagnostik erfolgen. Dazu zählt die Gastroösophagoduodenoskopie, ggf. auch mit Biopsieentnahmen zur histologischen Differenzierung. Bei typischen Refluxsymptomen und fehlendem Ansprechen auf eine empirische PPI-Therapie soll eine 24-Stunden-pHMetrie mit Impedanzmessung bevorzugt eingesetzt werden. Zur differenzialdiagnostischen Abgrenzung retrosternaler Beschwerden kann zudem eine Ösophagusmanometrie durchgeführt werden.

Andere Medikamente. Andere Medikamente (z.B. Antazida, $\mathrm{H}_{2}$-Rezeptorantagonisten) können bei GERD im Einzelfall auch eingesetzt werden, sind den PPI hinsichtlich der Wirksamkeit aber unterlegen [2].

\section{Tipp für die Praxis}

Eine Operation soll bei Patienten mit NERD und Therapieversagen nur dann erfolgen, wenn zweifelsfrei die Refluxgenese der persistierenden Symptome durch vollständige Funktionsdiagnostik belegt wurde [2].

\section{GERD in der Schwangerschaft}

Viele Frauen leiden in der Schwangerschaft unter gastrointestinalen Symptomen. Hierfür ist sehr häufig eine GERD verantwortlich. Die GERD-Prävalenz bei Schwangeren ist mit 40-85\% angegeben $[2,4,6]$. Eine Differenzierung zwischen einer GERD und leichteren, schwangerschaftsassoziierten gastrointestinalen Beschwerden wird nur selten vorgenommen. Die Erkrankung kann zu jedem Zeitpunkt in der Schwangerschaft auftreten und reicht von gelegentlichen, tolerablen Refluxbeschwerden bis hin zu gravierenden Symptomen und Veränderungen der Speiseröhre. In einer prospektiven Longitudinalstudie in Deutschland mit 510 Schwangeren klagten 26,1\% der Frauen im 1. Trimenon, $36,1 \% \mathrm{im} 2$. Trimenon und $51,2 \% \mathrm{im} 3$. Trimenon über GERD-Symptome. Eine medikamentöse Behandlung erfolgte bei $12,8 \%$ im $1 ., 9,1 \%$ im 2 . und $15,7 \%$ im 3. Trimenon [12].

Die Prävalenz von GERD in der Schwangerschaft liegt bei $40-85 \%$. 
Risikofaktoren. Untersuchungen zu korrelierenden Faktoren der Schwangerschaft und GERD erbrachten ein erhöhtes Erkrankungsrisiko während der Schwangerschaft mit steigendem Gestationsalter, der Parität und bereits vor der Schwangerschaft bestehenden Refluxbeschwerden sowie GERD in vorausgegangenen Schwangerschaften [6]. Kein Zusammenhang konnte gezeigt werden für Refluxkrankheit und BMI vor der Schwangerschaft und Gewichtszunahme in der Schwangerschaft $[4,6]$. Bezüglich ethnischer Unterschiede ist in der Literatur eine höhere Inzidenz von GERD bei Kaukasiern gegenüber Nigerianern (78,9 vs. $9 \%$ ) beschrieben worden, zwischen Europäern und Asiaten konnte kein Unterschied gefunden werden $[4,6]$.

\section{Pathophysiologie}

Die pathophysiologischen Ursachen für GERD in der Schwangerschaft werden kontrovers diskutiert. Die Annahme, dass die Refluxbeschwerden in der Schwangerschaft allein ausgelöst werden durch die intraabdominelle Druckerhöhung, bedingt durch den graviden Uterus, wurde im Jahre 1981 von Van Thiel u. Mitarbeitern widerlegt [13]. Es wurden verschiedene Untersuchungen an Patienten mit Aszites (Pseudogravidität) durchgeführt und es konnte nachgewiesen werden, dass bei diesen Patienten der Ösophagussphinktertonus nicht verändert war, es wurde sogar eine leichte Tonussteigerung festgestellt. Die Uterusgröße führt zudem erst in der 2. Schwangerschaftshälfte zu einer intraabdominellen Druckerhöhung, während GERDSymptome bereits häufig in der 1. Schwangerschaftshälfte auftreten [13]. Heute wissen wir, dass die Hauptursache für einen gastroösophagealen Reflux in der Schwangerschaft ein hormonell bedingt veränderter Tonus des unteren Ösophagussphinkters ist [4,6].

Im 1. Trimester ist der Ruhetonus des unteren Ösophagussphinkters noch nicht beeinträchtigt, jedoch ist seine Antwort auf physiologische Stimuli (z. B. Pentagastrin, proteinreiche Kost usw.) vermindert. Im Verlauf der Schwangerschaft kommt es durch den Einfluss von Progesteron und Östrogen bis zur 36. Schwangerschaftswoche zu einem zunehmend nachlassenden unteren Ösophagussphinktertonus $[4,6,14]$.

GERD in der Schwangerschaft beruht v. a. auf einem verringerten Tonus des unteren Ösophagussphinkters.
Es konnte wiederholt gezeigt werden, dass Progesteron im Zusammenspiel mit Östrogen für die Tonussenkung des Ösophagussphinkters verantwortlich ist. Östrogen ist hierbei ein notwendiger Primer für die Progesteronwirkung $[4,6,14]$.

Der hormonell bedingte niedrige untere Ösophagussphinktertonus in der Schwangerschaft kann dem steigenden intraabdominellen bzw. intragastrischen Druckanstieg durch den graviden Uterus funktionell nicht mit einem Tonusanstieg antworten $[3,4,13]$. Zudem begünstigt die hormonell bedingte Motilitätsstörung der Speiseröhre die Entstehung von GERD in der Schwangerschaft $[4,6,13]$. Durch die verminderte Peristaltik des Ösophagus kommt es zu einem verlängerten Kontakt von saurem Mageninhalt mit der Schleimhaut des Ösophagus. Neben Mageninhalt kann bei ebenso bestehender Tonussenkung des Pylorus auch ein Reflux von Galle in den Magen und konsekutiv in den unteren Ösophagus begünstigt werden $[4,15]$.

Fazit für die Praxis

Hauptursache der Refluxbeschwerden in der Schwangerschaft ist ein verminderter Tonus des unteren Ösophagussphinkters, bedingt durch physiologische Veränderungen im Hormonstatus.

Hierzu zeigten Untersuchungen mit 24-StundenpH-Metrien bei Schwangeren jedoch, dass Sodbrennen in der Schwangerschaft in erster Linie auf einen Reflux von saurem Mageninhalt beruht, ein alkalischer Reflux (Galle) lag nur selten vor. Gastroskopische Untersuchungen an schwangeren Frauen wiesen bisher nur gelegentlich entzündliche Veränderungen der Ösophagusschleimhaut nach $[4,6,13]$.

Die hormonell bedingte Motilitätsstörung des Ösophagus und die Tonussenkung des Ösophagussphinkters sowie die intraabdominelle Druckerhöhung infolge Uterusvergrößerung fallen nach der Entbindung weg, sodass Refluxbeschwerden und Sodbrennen oft schlagartig verschwinden [4].

Schon am 2. Tag post partum normalisiert sich der untere Ösophagussphinktertonus [4,6]. 


\section{Klinische Manifestation}

\section{—ösophageale Symptomatik}

Die Verteilung typischer GERD-Symptome in der Schwangerschaft ist ähnlich wie bei nicht schwangeren Patienten $[4,6,12]$. Lediglich die Regurgitation des sauren Mageninhalts tritt in der Schwangerschaft signifikant häufiger auf $[4,6]$. Sodbrennen ist jedoch, wie außerhalb der Schwangerschaft, das häufigste Symptom und nimmt zudem mit Fortschreiten der Schwangerschaft zu $[4,12]$. Sodbrennen kann sich klinisch mit teils schmerzhaften, teils krampfartigen Beschwerden von unterschiedlicher Intensität und Dauer präsentieren, das spontan oder in $77 \%$ im Zusammenhang mit der Nahrungsaufnahme auftreten kann [6]. Postprandiale Beschwerden können so stark sein, dass Patientinnen nur eine Mahlzeit pro Tag zu sich nehmen und in sitzender Position versuchen zu schlafen, um Symptome zu lindern oder zu vermeiden.

Die Symptome von GERD in und außerhalb der Schwangerschaft präsentieren sich gleichermaßen.

Durch GERD bedingte Komplikationen wie Ösophagitis, Strikturen oder Blutungen finden sich in der Schwangerschaft nur selten. Man geht davon aus, dass hierfür eine längere Erkrankungsdauer einer GERD vorliegen muss. In endoskopischen Untersuchungen stellte man fest, dass $2 / 3$ der an Reflux leidenden Schwangeren histologisch eine Ösophagitis aufwiesen, die sich endoskopisch aber nur als eine milde Form erwies. Ösophageale Erosionen zeigen sich bei Schwangeren höchst selten $[4,6,16]$.

Eine prospektive Studie aus Deutschland zeigte eine massive Beeinträchtigung der Lebensqualität schwangerer Frauen durch GERD [12].

GERD führt in der Schwangerschaft zu einer massiven Beeinträchtigung der Lebensqualität.

\section{Extraösophageale Symptome}

Extraösophageale Symptome wie Heiserkeit, chronischer Husten, chronische Laryngitis und Asthma sind bei Nicht-GERD-Patienten gut untersucht und treten durchaus häufig auf. Zu extraösophagealen Symptomen in der Schwangerschaft gibt es jedoch nur eine Arbeit. In dieser Studie wurden bei 210 schwangeren Frauen extraösophageale Symptome erfragt und es zeigte sich, dass diese in der Schwangerschaft kaum auftreten. Auch hinsichtlich des Ausbleibens der extraösophagealen Symptome wird die geringe zeitliche Dauer einer Schwangerschaft diskutiert [17].

\section{Diagnostik in der Schwangerschaft}

Als Goldstandard für die Diagnosestellung gilt eigentlich die 24-Stunden-pH-Metrie, die jedoch aufgrund der Belastung für den Patienten und der limitierten Verfügbarkeit nur bei speziellen Fragestellungen angewandt wird. Daher wird GERD bei schwangeren Frauen meist anamnestisch durch die genaue Erhebung der Symptome und ihrer Häufigkeit diagnostiziert. Hierfür stehen standardisierte validierte Fragebögen zur Verfügung.

Bei dem Verdacht auf Komplikationen der GERD ist die Gastroösophagoduodenoskopie indiziert $[4,16]$.

\section{Tipp für die Praxis}

Endoskopische Untersuchungen können bei gutem Monitoring von Blutdruck, Sauerstoffsättigung, fetalem Monitoring und angepasster Dosierung von zugelassenen Sedativa sicher und ohne Schäden für Mutter und Kind durchgeführt werden.

Bisher wurden die meisten diagnostischen Studien zur Refluxkrankheit in der Schwangerschaft mit pH-Monitoring, Langzeit-pH-Metrien, Manometrien, Hormonspiegelmessungen und nur selten endoskopisch durchgeführt [4,13-15].

\section{Therapie}

Sowohl Schwangere als auch die behandelnden Ärzte betrachten gastrointestinale Beschwerden in der Schwangerschaft häufig als normal, vorübergehend und folglich als zu akzeptierendes „Übel“. Es gibt viele, oft pseudomedizinische Erklärungen für gastrointestinale Symptome und je nach Leidensdruck werden sie meist als gegeben hingenommen.

Zwischen einer Emesis gravidarum, Unwohlsein, Bevorzugung bestimmter Speisen und einer echten Refluxkrankheit bestehen fließende Übergänge, was eine Therapie nicht immer einfach macht. Und sicherlich handelt es sich nicht immer um eine GERD, wenn Schwangere über gastrointestinale Beschwerden im 1. Trimenon klagen. Aber die Prävalenz der GERD ist in 
der Schwangerschaft hoch, und die Patientinnen würden von einer spezifischen Behandlung profitieren.

Jeder behandelnde Arzt, unabhängig ob Gynäkologe, Allgemeinmediziner oder Gastroenterologe, sollte nach GERD-Symptomen in der Schwangerschaft fragen und diese auch behandeln.

An erster Stelle des Behandlungsansatzes stehen Verhaltensänderungen und eine Regulierung der Nahrungsaufnahme und der Darmtätigkeit.

Wenn Maßnahmen der Lebensstil-Änderung nicht greifen, sollte nach dem unten beschriebenen Stufenkonzept medikamentös therapiert werden.

\section{Cave}

Vor einer medikamentösen Therapie muss eine ausführliche Medikamentenanamnese durchgeführt werden. Medikamente können GERD verursachen, eine vorbestehende GERD-Symptomatik durch die Beeinflussung der Motilität verstärken, zu einer Relaxation des unteren Ösophagussphinkters führen oder die ösophageale Clearance stören.

\section{Tabelle 1}

Ernährung und GERD in der Schwangerschaft (nach [4]).

\begin{tabular}{|lll|}
\hline Speisen & Zielorgan & Wirkung \\
\hline fetthaltige Speisen & Ösophagussphinkter & Tonusminderung \\
\hline Kaffee & Magen & erhöht die Säureproduktion \\
\hline Alkohol & Magen & erhöht die Säureproduktion \\
\hline Süßigkeiten & Magen & erhöhen die Säureproduktion \\
\hline Süßigkeiten & Ösophagussphinkter & Tonusminderung \\
\hline zu stark gewürzte Speisen & Magen & erhöhen die Säureproduktion \\
\hline Tomaten & Magen & erhöhen die Säureproduktion \\
\hline Pfefferminze & Ösophagus & enthalten zusätzliche Säure \\
\hline Lüurehaltige Obstsäfte & Ösophagus & hoher Säuregehalt die Säureproduktion \\
\hline kitrusfrüchte & Magen & wirken blähend \\
\hline
\end{tabular}

\section{Medikamentöse Therapie}

Aus neueren Studiendaten ist bekannt, dass schwangere Patientinnen mit GERD trotz massiver Einschränkung der Lebensqualität nicht adäquat medikamentös therapiert werden $[5,12]$. Gründe dafür sind v.a. die meist vorherrschende Unsicherheit der behandelnden Ärzte hinsichtlich medikamentöser Möglichkeiten in der Schwangerschaft sowie oft auch die Angst der Schwangeren vor fetaler Schädigung.

Medikamente sind in der Schwangerschaft grundsätzlich nur nach strenger Nutzen-RisikoAbwägung einzusetzen.

Ist jedoch durch ein Krankheitsbild die Lebensqualität der Frau beeinträchtigt, sollte die Patientin medikamentös behandelt werden. Vor Beginn der Therapie muss eine ausführliche Aufklärung über Nutzen und Risiko sowie therapeutische Alternativen erfolgen. In der Schwangerschaft sollte leitliniengerecht therapiert 
werden. Das empfohlene medikamentöse Therapieschema lautet: Antazida/Sucralfat - H2-RA (Histamin2-Rezeptorantagonisten) - PPI (ProtonenpumpenInhibitoren) $[2,4,6]$.

Antazida zeigen keine relevanten Effekte auf das ungeborene Kind und sind demnach Mittel der Wahl zur primären Behandlung von GERD in der Schwangerschaft.

Für H2-RA liegen umfangreiche Erfahrungen im klinischen Alltag wie auch in Fallkontrollstudien vor, die keinen Hinweis auf ein erhöhtes teratogenes Risiko zeigen [18].

PPI werden generell mit großer Zurückhaltung in der Schwangerschaft verordnet. Es steht mittlerweile aber eine Reihe an wissenschaftlichen Daten zur Verfügung, welche den Einsatz von PPI in der Schwangerschaft zulassen. Entsprechend der neuen S2k-Leitlinie sind PPI bei therapieresistenten persistierenden GERD-Symptomen empfohlen [2]. Ebenso empfiehlt die aktuelle S2k-Leitlinie, eine wirksame Therapie in der Schwangerschaft mit einem H2-Rezeptorantagonisten oder einem PPI bei klinischem Bedarf fortzuführen [2].

\section{Tipp für die Praxis}

GERD sollte in der Schwangerschaft nach einem Stepup-Algorithmus therapiert werden: Allgemeinmaßnahmen - Antazida/Sucralfat - H2-RA (Histamin-2Rezeptorantagonisten) - PPI (Protonenpumpeninhibitoren).

\section{Antazida}

Antazida gelten als Mittel der Wahl zur primären medikamentösen Behandlung von GERD in der Schwangerschaft. Antazida wirken neutralisierend auf die Magensäure, ohne deren Produktion zu beeinflussen. Sie wirken lokal, schnell und effektiv und werden nur in sehr geringen Spuren systemisch resorbiert $[4,6]$. Die Einnahme von magnesium- und kalziumhaltigen Antazida während der Schwangerschaft ist in einem europäischen Konsens bezüglich der teratogenen Wirkung als unbedenklich eingestuft worden [19]. Auch in den aktuellen Leitlinien von 2014 gelten Antazida als Erstlinien-Therapie in der medikamentösen Behandlung von GERD in der Schwangerschaft. Kalziumhaltige Antazida zeigen zudem einen positiven Effekt auf die Vermeidung von Hypertension und Präeklampsie in der Schwangerschaft. Auch bei Zufuhr von Magnesiumsulfat konnte in einer großen, randomisierten Stu-

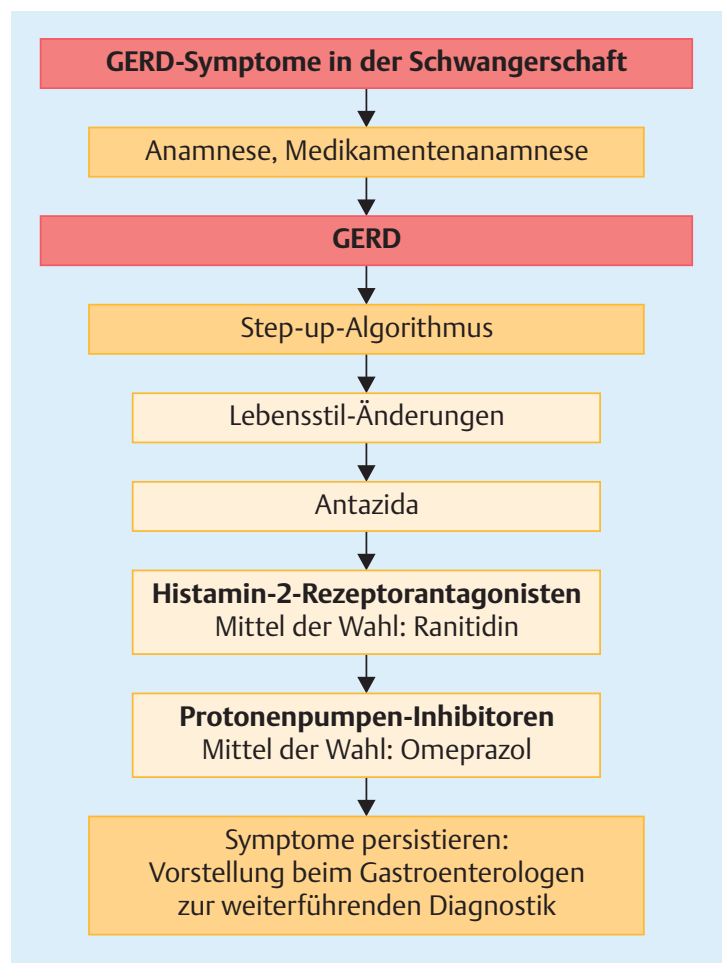

Abb. 2 Diagnostik und Therapie von GERD in der Schwangerschaft (nach [4]).

die ein um 50\% reduziertes Risiko für Eklampsie gegenüber Placebo festgestellt werden, nennenswerte Nebenwirkungen traten nicht auf [20].

\section{Sucralfat}

Sucralfat ist ein Aluminiumsalz des Saccharosesulfats, welches nur minimal systemisch resorbiert wird. Der Sucralfat-Protein-Komplex bildet einen Schutzfilm und bedeckt die Magenschleimhaut. Zudem werden Pepsin und Gallensäuren gebunden.

Sucralfat ist das einzige nichtresorbierbare Medikament, welches in randomisierten kontrollierten Studien während der Schwangerschaft untersucht wurde. In einer Studie wurde die Effektivität von Sucralfat vs. Änderungen im Lebensstil bei GERD in der Schwangerschaft untersucht. Die Remissionsrate war deutlich höher bei Schwangeren, die mit Sucralfat therapiert wurden, als bei Frauen, die nicht medikamentös therapiert worden waren.

In tierexperimentellen Studien konnte gezeigt werden, dass die Einnahme von Sucralfat weder einen Einfluss auf die Fertilität hat, noch wurden fetale Missbildungen 
beobachtet. Die getestete Dosierung lag 50-fach höher, als sie beim Menschen üblicherweise angewendet wird. Eine exzessive Aluminiumanreicherung kann eine Zunahme perinataler Mortalität, Osteopenie und Einfluss auf Merk- und Lernfähigkeit fördern. Es ist aber darauf hinzuweisen, dass dieses Risiko nur bei bestehender Nierenerkrankung und nur nach Einnahme höherer Dosen über einen längeren Zeitraum existiert und somit bei Frauen ohne Nierenerkrankung keine Auswirkung hat [4].

\section{Histamin-2-Rezeptorantagonisten (H2-RA)}

H2-RA wirken systemisch. Ranitidin ist der am häufigsten verordnete H2-RA bei GERD in der Schwangerschaft. Ranitidin ist bisher der einzige Histamin-2Rezeptorantagonist mit nachgewiesener Effektivität in der Schwangerschaft.

Bei GERD in der Schwangerschaft gilt Ranitidin als Mittel der ersten Wahl.

Studienlage. In einer doppelblinden, placebokontrollierten, dreifachen Cross-over-Studie wurde Ranitidin über 4 Wochen schwangeren Frauen verabreicht, bei denen Lebensstil-Änderungen und Antazida zu keiner Besserung geführt hatten. Gegenüber Placebo führte Ranitidin zu einer deutlichen Reduktion der Symptome; Nebenwirkungen wurden keine beschrieben [18].

Eine Beobachtungsstudie aus den Jahren 1985-1992 zeigte, dass Frauen unter Einnahme von Ranitidin sowie Cimetidin eine Inzidenz der fetalen Malformationsrate aufwiesen, die einer schwangeren Population ohne Medikamenteneinfluss in der Schwangerschaft entspricht [21]. In einer anderen prospektiven Kohortenstudie aus dem Jahre 1996 wurde beschrieben, dass der Einsatz von Ranitidin, Cimetidin, Famotidin und Nizatidin in der Schwangerschaft keine Nebenwirkungen bzw. Auswirkungen auf Feten zeigte [22]. In der Michigan-Medicaid-Beobachtungsstudie zeigten aber 2 von 33 famotidinexponierten Feten (im 1. Trimester) schwere Malformationen. Aufgrund der kleinen Fallzahl kann dazu aber keine Aussage gemacht werden [23].

Für das Medikament Nizatidin gibt es widersprüchliche Daten zum Einsatz in der Schwangerschaft. In tierexperimentellen Untersuchungen wurde eine erhöhte Abortrate, ein geringeres Geburtsgewicht und eine höhere Rate von tot geborenen Feten angegeben $[4,6]$.
Diese Ergebnisse konnten jedoch in anderen Studien nicht bestätigt werden $[4,6]$.

Cimetidin wiederum zeigte in tierexperimentellen Studien eine antiandrogene Wirkung mit Veränderungen im Bereich der Hoden, Prostata und Samenbläschen. Eine antiandrogene Wirkung konnte bei keinem der anderen H2-RA nachgewiesen werden $[4,6,23]$.

\section{Cave}

Die Anwendung von Cimetidin in der Schwangerschaft wird aufgrund der in tierexperimentellen Studien und bei Nichtschwangeren beobachteten Feminisierung nicht angeraten [19].

\section{Protonenpumpen-Inhibitoren (PPI)}

Protonenpumpen-Inhibitoren sind die wirkungsvollsten Medikamente gegen GERD und Ösophagitis. PPI wurden bei Schwangeren bislang relativ zurückhaltend eingesetzt, wobei es mittlerweile genügend prospektive und retrospektive Studienergebnissen zu PPI in der Schwangerschaft gibt, die den Einsatz von PPI in der Schwangerschaft bei komplizierter GERD vertreten.

Noch im Jahre 2003 wurde in einem europäischen Konsensusmeeting in der Schweiz, bei dem Experten aus dem Gebiet der Gastroenterologie, der Geburtshilfe und der Pharmazie teilnahmen, der Einsatz von PPI in der Schwangerschaft nicht empfohlen [19]. Die damals durchgeführte Untersuchung zum Einsatz von Omeprazol zeigte eine erhöhte embryonale und fetale Mortalität in tierexperimentellen Studien. Zudem erhielt die FDA Berichte über schwere fetale Malformationen (Anenzephalie und Hydroenzephalie) unter Einnahme von Omeprazol in der Schwangerschaft [23]. Andere Fallbeschreibungen vor 2003 zeigten hingegen keine kongenitalen fetalen Fehlbildungen bei Schwangeren unter PPI-Einnahme [4,6].

In der aktuellen S2k-Leitlinie der Gesellschaft für Verdauungs- und Stoffwechselkrankheiten wurde der Einsatz von PPI aufgrund folgender Studienergebnisse in der Schwangerschaft mit einem starken Konsens empfohlen [2]:

Die aktuelle S2k-Leitlinie 2014 zur Therapie von GERD empfiehlt den Einsatz von PPI in der Schwangerschaft bei persistierenden Beschwerden unter H2-RA. 
Studienlage. In einer Metaanalyse von 7 Studien (1530 PPI-Konsumenten und 133410 Kontrollen ohne PPIEinnahme) fanden sich keine Hinweise auf relevante Schädigungen des Kindes, eine erhöhte Rate an Frühgeborenen oder Aborten [24]. In einer anderen Metaanalyse wurden 5 Kohortenstudien analysiert, um das Risiko kongenitaler Fehlbildungen bei PPI-Einnahme im 1. Trimester einzuschätzen [25]. Die meisten Schwangeren erhielten Omeprazol (534 von 593), und die Fehlbildungsrate entsprach mit 1,18\% einem Normalkollektiv ohne Medikamenteneinnahme [25].

In einer großen dänischen Kohortenstudie wurde bei 5082 von 840968 Lebendgeborenen eine PPI-Exposition während der Schwangerschaft bzw. in den 4 Wochen vor der Konzeption registriert. PPI-Einnahme während des 1 . Trimenons war nicht mit relevanten Fehlbildungen assoziiert. Auffällig war aber in der dänischen Studie eine Risikoerhöhung bei PPI-Einnahme in den letzten 4 Wochen vor der Konzeption dies traf allerdings nicht für Omeprazol zu.

\section{Tipp für die Praxis}

Frauen, die eine Konzeption planen und einen PPI benötigen, sollte Omeprazol verordnet werden [26,27].

Eine weitere große Fallkontrollstudie aus Israel mit 1186 PPI-Expositionen während des 1. Trimenons ergab ebenfalls keinen Hinweis auf eine erhöhte Fehlbildungsrate, ebenso wirkte sich eine PPI-Einnahme im 3. Trimenon nicht negativ aus im Hinblick auf Frühgeburten, perinatale Morbidität und Mortalität sowie niedriges Geburtsgewicht [28].

In tierexperimentellen Studien wurde zudem der PPI Lansoprazol in 40-fach höherer Wirkstoffdosierung als beim Menschen untersucht. Lansoprazol zeigte keinen Einfluss auf die Fertilität und keine Schädigung des Feten [29]. Für den Einsatz von Pantoprazol, Rabeprazol und Esomeprazol in der Schwangerschaft liegen bisher nur wenige Daten vor. 2005 wurde eine Multizenterstudie veröffentlicht, die zeigte, dass die neueren PPI nicht mit einem erhöhten teratogenen Risiko vergesellschaftet sind. Laut Herstellerangaben erwiesen sich die neueren PPI auch in Tierversuchen als sicher [23].

Zusammenfassend scheinen die PPI in der Schwangerschaft als sicher zu gelten.

\section{Tipp für die Praxis}

Die Indikation von PPI im 1. Trimester sollte trotz der Daten zur Sicherheit kritisch streng gestellt werden, um eine Teratogenität auszuschließen.

Die Empfehlungen der Hersteller einzelner PPI im Hinblick auf eine Anwendung in der Schwangerschaft lauten:

- Esomeprazol nur mit Vorsicht

- Lansoprazol nicht empfohlen

- Omeprazol nur nach sorgfältiger Nutzen-RisikoAbwägung

- Pantoprazol: Kontraindikation

- Rabeprazol: Kontraindikation [2,6,30]

\section{GERD in der Laktationsphase}

Die Symptome einer GERD verschwinden bei den meisten Frauen nach der Geburt schnell [4,6,31]. Wenn GERD-Beschwerden postpartal fortbestehen, ist eine medikamentöse Therapie indiziert.

Bei stillenden Frauen gilt der bereits für die Zeit der Schwangerschaft benannte Step-up-Algorithmus.

Bei nicht stillenden Müttern kann nach dem beschrieben Step-down-Schema zur Therapie der GERD außerhalb der Schwangerschaft begonnen werden. Hier kommen primär PPI zum Einsatz.

Übergang in die Muttermilch. In der Laktationsphase ist es wichtig zu beachten, dass alle systemisch wirkenden Anti-Reflux-Mittel in die Muttermilch übertreten (Ausnahme: Aluminium- und Magnesiumhydroxid) und somit Einfluss auf den Säugling haben könnten. Alle eventuellen therapeutischen Optionen müssen demnach mit der stillenden Mutter ausführlich besprochen werden und das jeweilige Nutzen-RisikoProfil abgewogen werden [23].

Antazida. Während der Stillzeit gelten Antazida als sicheres Medikament für den Säugling.

Antazida können in der Laktationsphase bedenkenlos eingesetzt werden.

H2-RA. Histamin-2-Rezeptorantagonisten werden nachweislich mit der Muttermilch ausgeschieden. Cimetidin und Ranitidin erreichen in der Muttermilch eine 4- bis 7-fach höhere Konzentration als im mütter- 
Kasuistik zum fallorientierten Lernen

Eine 28-jährige I Gravida, 0 Para stellt sich in der gynäkologischen Ambulanz der Universitätsfrauenklinik Regensburg in der 20. + 5 SSW zur regulären Schwangerschaftsvorsorgeuntersuchung vor. Die Schwangerschaft war bisher komplikationslos verlaufen. Die Patientin klagte jedoch über zunehmendes Sodbrennen. Sodbrennen kannte die Patientin bereits seit mehreren Jahren. Die Patientin bemerkte die Symptomatik bisher meist in Verbindung mit Stresssituationen. Eine Diagnostik erfolgte vor ca. 9 Monaten. Bei endoskopisch nachgewiesener Helicobacter-pylori-negativer Antrumgastritis wurde eine Therapie mit PPI begonnen. Die Patientin äußerte Sorgen bezüglich der PPI-Therapie in der präkonzeptionellen Phase und der Frühgravidität. Sie und ihr Ehemann haben Angst, dass die Therapie in der Frühschwangerschaft einen negativen Einfluss auf das ungeborene Kind hat. Die Medikation hatte die Patientin deshalb in der 9. SSW abgebrochen.

\section{Anamnese}

In der Anamnese fanden sich keine sonstigen Erkrankungen; die Familienanamnese war unauffällig.

Die Patientin nimmt seit bekannter Schwangerschaft Folsäure ein. Es bestehen keine bekannten Allergien. Die Patientin hatte über mehrere Jahre ein orales Kontrazeptivum eingenommen. Die Medikamentenanamnese war bis auf die PPI-

Therapie leer. Die Patientin nahm $20 \mathrm{mg}$ Omeprazol 1-0-0 bereits 2 Monate präkonzeptionell sowie die ersten 9 SSW ein.
Der bisherige Schwangerschaftsverlauf war unauffällig. Die Schwangerschaft war spontan entstanden. In den regulären Ultraschalluntersuchungen zeigte sich bisher ein zeitgerecht entwickelter männlicher Fet in Schädellage. Alle erhobenen Laborwerte lagen im Normbereich. Das Sodbrennen war seit der 17. SSW wieder aufgetreten.

Die Patientin berichtet über saures Aufstoßen sowie starkes Brennen hinter dem Brustbein an mindestens 3 Wochentagen. Zudem gibt sie an, dass Einschlafen und Durchschlafen durch das Brennen beeinträchtigt sind. Auch die Nahrungsaufnahme sei durch die belastenden Symptome deutlich gestört. Auf Nachfragen berichtet die Patientin über aktuell psychischen Stress, da ein Wohnungsumzug in 2 Wochen geplant ist.

\section{Therapie}

Aufgrund der bereits vor der Schwangerschaft diagnostizierten Gastritis erfolgte präkonzeptionell sowie in der Frühgravidität eine PPI-Therapie. Die Therapie wurde von der Patientin aus Angst vor Schädigung des Kindes bei Bekanntwerden der Schwangerschaft abgebrochen. Aufgrund der erneut aufgetretenen Symptomatik mit der Verdachtsdiagnose einer GERD musste ein Therapieschema entsprechend dem empfohlenen Step-upAlgorithmus mit der Patientin besprochen werden. Vor erneutem Beginn der medikamentösen Therapie wurde die Patientin über mögliche Lebensstil-Änderungen sowie Änderungen im Essverhalten zur Prävention von GERD aufgeklärt. Da die Patientin die Symptomatik schon kannte, hatte sie bereits eigenständig die Ernährung und Lebensgewohnheiten umgestellt.
Die Gewichtszunahme seit Beginn der Schwangerschaft lag im unteren Normbereich. Es erfolgte eine ausführliche Aufklärung der Patientin über die aktuelle Studienlage zum Einsatz von Medikamenten für die Behandlung von GERD in der Schwangerschaft. Im Rahmen dieses Gesprächs konnte der Patientin die Sorge bezüglich einer Omeprazol-Einnahme in der präkonzeptionellen Phase sowie der Frühgravidität genommen werden. Eine weiterführende Diagnostik wurde von der Patientin trotz Aufklärung abgelehnt.

Somit erfolgte der Beginn der Therapie mit Ranitidin (150 mg). Eine Wiedervorstellung wurde in 2 Wochen vereinbart. Der Patientin wurde angeraten, zwischenzeitlich einen fetalen Organultraschall durchführen zu lassen.

Bei der Wiedervorstellung der Patientin hatte sich die GERD-Symptomatik nur mäßig gebessert. Der Ultraschall des Feten hatte einen unauffälligen Befund ergeben. In der 23. SSW wurde mit Einverständnis der Patientin mit einer erneuten PPI-Therapie (Omeprazol $20 \mathrm{mg}$ ) begonnen. Darunter war die Patientin bereits nach 3 Tagen beschwerdefrei. Aufgrund der neuen S2k-Leitlinien zu GERD von 2014 konnte der Patientin angeraten werden, die PPI-Therapie fortzuführen. Die Patientin wurde in der 39. SSW von einem lebensfrischen, gesunden, normalgewichtigen, männlichen Neugeborenen spontan entbunden. Postpartal erfolgte ein Auslassversuch der PPI-Therapie. Bei Beschwerdefreiheit konnte auf eine erneute medikamentöse Therapie in der Stillzeit verzichtet werden. Als Bedarfsmedikation wurde der Patientin Cimetidin empfohlen. lichen Serum. Famitidin erreicht ca. 6 Stunden nach Einnahme eine mittlere Milch-Plasma-Konzentration von $1,78[4,6]$.

Nizatidin wird nur in sehr geringen Konzentrationen mit der Muttermilch ausgeschieden. In einer tierexperimentellen Studie zeigte sich jedoch eine kindliche
Wachstumsretardierung unter Einnahme von Nizatidin $[4,6]$.

Die „American Academy of Pediatrics“ hat Cimetidin im Jahre 1994 als ein für die Einnahme während der Laktationsphase sicheres Medikament erklärt. Auch Ranitidin und Famotidin werden als unschädlich für den Säugling betrachtet, nur Nizatidin sollte aufgrund der 
Ergebnisse der tierexperimentellen Studien vermieden werden $[4,6]$.

PPI. Leider gibt es zur Einnahme von Protonenpumpeninhibitoren in der Laktationsphase und deren Einfluss auf das Neugeborene nur eine sehr dünne Datenlage. Aufgrund des niedrigen Molekulargewichts der PPI wird jedoch angenommen, dass sie in die Muttermilch übertreten. Es zeigte sich 3 Stunden nach Einnahme von Omeprazol eine Konzentration von Omeprazol in der Muttermilch, die 7\% unter dem Wert liegt, der 4 Stunden nach Einnahme im Serum der Mutter nachweisbar ist [27]. Es wird wohl nur eine geringe Menge an Omeprazol am Säugling zur Wirkung kommen. Leider gibt es bisher nur Daten aus Einzelfalluntersuchungen. Unter Einnahme von Omeprazol und Lansoprazol während der Laktation zeigte sich in tierexperimentellen Untersuchungen ein vermindertes Körpergewicht der Rattenbabys [23].

PPI sind während der Stillzeit nicht empfohlen [27].

\section{Kernaussagen}

Die gastroösophageale Refluxkrankheit ist eine sehr häufige Erkrankung in der Schwangerschaft, welche die Lebensqualität der schwangeren Frau massiv beeinträchtigen kann. Die Symptome der gastroösophagealen Refluxkrankheit in der Schwangerschaft unterscheiden sich kaum von den klassischen Symptomen der GERD bei „Nichtschwangeren“. Aufgrund der weit verbreiteten Meinung, dass gastrointestinale Symptome in der Schwangerschaft normal sind, ist anzunehmen, dass die Dunkelziffer manifester Refluxkrankheiten höher ist als bislang beschrieben.

Die Folgen einer unbehandelten GERD sind bisher nicht bekannt. Man weiß jedoch, dass schwangere Frauen mit GERD bisher nicht adäquat therapiert werden. Die extraösophagealen Symptome in der Schwangerschaft sind aufgrund des relativ kurzen Belastungszeitraums deutlich geringer als in der Nichtschwangeren-Bevölkerung.

Laut der neuen S2k-Leitlinie von 2014 ist weiterhin der Step-up-Algorithmus empfohlen. Beginnend mit Lebensstil-Änderungen, gefolgt von Antazida, H2-RA sowie Protonenpumpen-Inhibitoren. Es gibt viele neue Daten zum sicheren Einsatz von PPI in der Schwangerschaft, somit enthält die neue Leitlinie die Empfehlung des Einsatzes von PPI bei symptombelasteter Frau in der Schwangerschaft.

Für einen genaueren Einblick in die Zusammenhänge von GERD und dessen Symptomen in der Schwangerschaft benötigt man jedoch neuere Studien und Daten, die die Häufigkeit, die Stärke der Symptome und die Lebensqualität erfassen. GERD muss als Differenzialdiagnose bei gastrointestinalen Beschwerden in der Schwangerschaft immer mit aufgelistet werden. Die Diagnose kann dann anhand der Symptome gestellt werden und durch eine Therapieoptimierung die Lebensqualität der Schwangeren frühzeitig verbessert werden. Zudem werden dringend Studiendaten zu GERD und die medikamentöse Therapie in der Laktationsphase benötigt, um auch dahingehend neue Leitlinien etablieren zu können. 


\section{Über die Autoren}

\section{Sara Fill Malfertheiner}

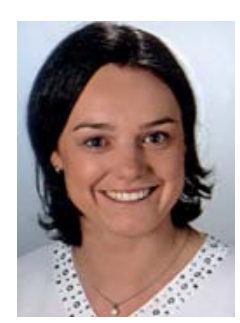

Dr. med. MHBA. Jahrgang 1980.

1999-2004 Studium der Humanmedizin an der Leopold Franzens Universität Innsbruck, Österreich. 2004 Approbation und Promotion an der Universität Innsbruck. 2005 Promotion an der Universität Bologna, Italien. 2005-2010 wissenschaftliche Mitarbeiterin der Universitätsfrauenklinik Magdeburg. 2006-2007 Forschungsstipendiatin an der Yale University, USA. 2009-2012 berufsbegleitendes Studium mit dem Abschluss: Master of Health in Business Administration (MHBA) an der FriedrichAlexander-Universität Erlangen-Nürnberg. Seit 2013 Assistenzärztin an der Universitätsfrauenklinik/Barmherzige Brüder-St. Hedwig, Regensburg. Wissenschaftlicher Schwerpunkt: klinische Forschung im Bereich gastrointestinale Erkrankungen in der Schwangerschaft, Grundlagenforschung des Mikrobioms in der Schwangerschaft und Perinatalperiode.

\section{Korrespondenzadresse}

Dr. med. MHBA Sara Fill Malfertheiner

Universitätsfrauenklinik Regensburg

Klinik St. Hedwig

Steinmetzstraße 1

93049 Regensburg

E-Mail: sara.fill-malfertheiner@barmherzige-regensburg.de

\section{Interessenkonflikt}

Die Autorinnen erklären, dass kein Interessenkonflikt besteht. 


\section{Literatur}

1 Katz PO, Gerson LB, Vela MF. Guidelines for the diagnosis and management of gastroesophageal reflux disease. Am J Gastroenterol 2013; 108: $308-328$

2 Koop H, Fuchs KH, Labenz J et al. S2k-Leitlinie: Gastroösophageale Refluxkrankheit. Z Gastroenterol 2014; 52: 1299-1346

3 Vakil N, van Zanten SV, Kahrilas P et al.; Global Consensus Group. The Montreal definition and classification of gastroesophageal reflux disease: a global evidence-based consensus. Am J Gastroenterol 2006; 101: 1900-1920

4 Fill S, Malfertheiner M, Costa SD et al. [Handling of the gastroesophageal reflux disease (GERD) during pregnancy-a review. Z Geburtshilfe Neonatol 2007; 211: 215-223

5 Fill Malfertheiner S, Malfertheiner MV, Monkemuller K et al. Gastroesophageal reflux disease and management in advanced pregnancy: a prospective survey. Digestion 2009; 79: 115-120

6 Richter JE. Review article: the management of heartburn in pregnancy. Aliment Pharmacol Ther 2005; 22: 749-757

7 Ronkainen J, Aro P, Storskrubb T et al. High prevalence of gastroesophageal reflux symptoms and esophagitis with or without symptoms in the general adult Swedish population: a Kalixanda study report. Scand J Gastroenterol 2005; 40: 275-285

8 Fass R, Quan SF, O'Connor GT et al. Predictors of heartburn during sleep in a large prospective cohort study. Chest 2005; 127: 1658 1666

9 Kulig M, Nocon M, Vieth M et al. Risk factors of gastroesophageal reflux disease: methodology and first epidemiological results of the ProGERD study. J Clin Epidemiol 2004; 57: 580-589

10 Fass R, Achem SR. Noncardiac chest pain: epidemiology, natural course and pathogenesis. J Neurogastroenterol Motil 2011; 17 : $110-123$

11 Zagari RM, Fuccio L, Wallander MA et al. Gastro-oesophageal reflux symptoms, oesophagitis and Barrett's oesophagus in the general population: the Loiano-Monghidoro study. Gut 2008; 57 : 1354-1359

12 Fill Malfertheiner S, Malfertheiner MV, Kropf S et al. A prospective longitudinal cohort study: evolution of GERD symptoms during the course of pregnancy. BMC Gastroenterol 2012; 12: 131

13 Van Thiel DH, Wald A. Evidence refuting a role for increased abdominal pressure in the pathogenesis of the heartburn associated with pregnancy. Am J Obstet Gynecol 1981; 140: 420-422

14 Alvarez-Sanches A, Rey E, Achem SR et al. Does progesterone fluctuation across the menstrual cycle predispose to gastrooesophageal reflux? Am J Gastroenterol 1999; 94: 1468-1471

15 Schade RR, Pelekans MJ, TauxeWN et al. Gastric emptying during pregnancy. Gastroenterology 1984; 86: 1234A
16 Capell MS. The safety and efficacy of gastrointestinal endoscopy during pregnancy. Gastroenterol Clin North Am 1998; 27: 37-71

17 Malfertheiner M, Malfertheiner P, Costa SD et al. Extraesophageal symptoms of gastroesophageal reflux disease during pregnancy. Z Gastroenterol 2015; [accepted for publication]

18 Larson JD, Patatanian E, Miner PB et al. Double-blind, placebocontrolled study of ranitidine for gastroesophageal reflux symptoms during pregnancy. Obstet Gynecol 1997; 90: 83-87

19 Tytgat GN, Heading RC, Muller-Lissner S et al. Contemporary understanding and management of reflux and constipation in the general population and pregnancy: a consensus meeting. Aliment Pharmacol Ther 2003; 18: 291-301

20 Lindow SW, Regnell P, Sykes J et al. An open-label, multicentre study to assess the safety and efficacy of a novel reflux suppressant (Gaviscon Advance) in the treatment of heartburn during pregnancy. Int J Clin Pract 2003; 57: 175-179

21 Briggs GG, Freeman RY, Yaffe SJ. Drugs in Pregnancy and Lactation: a Reference Guide to fetal and neonatal Risk. Baltimore, USA: William and Wilkins; 2002

22 Finkelstein W, Isselbacker KJ. Cimetidine. N Engl J Med 1978; 229: 992-996

23 Magee LA, Inocencian G, Kamboijt R et al. Safety of first trimester exposure to histamine $\mathrm{H} 2$ blockers. A prospective cohort study. Dig Dis Sci 1996; 41: 1145-1149

24 Gill SK, O’Brien L, Einarson TR et al. The safety of proton pump inhibitors (PPIs) in pregnancy: a meta-analysis. Am J Gastroenterol 2009; 104: 1541 -1545

25 Nikfar S, Abdollahi M, Moretti ME et al. Use of proton pump inhibitors during pregnancy and rates of major malformations. A meta-analysis. Dig Dis Sci 2002; 47: 1526-1529

26 Pasternak B, Hviid A. Use of proton-pump inhibitors in early pregnancy and the risk of birth defects. N Engl J Med 2010; 363: 2114-2123

27 Majithia R, David A. Are proton pump inhibitors safe during pregnancy and lactation? Evidence to Date. Drugs 2012; 72: 171 -179

28 Matok I, Levy A, Wiznitzer A et al. The safety of fetal exposure to proton-pump inhibitors during pregnancy. Dig Dis Sci 2012; 57 : $699-705$

29 Anonymous. Prevacid (product information). Lake Forest, IL, USA: TAP Pharmaceutical; 2005

30 Mitchell AA. Proton-pump inhibitors and birth defects - some reassurance, but more needed. N Engl J Med 2010; 363: 2161 2163

31 Rey E, Rodriguez-Artalejo F, Herraiz MA et al. Gastroesophageal reflux symptoms during and after pregnancy: a longitudinal study. Am J Gastroenterol 2007; 102: 2395-2400 


\section{CME•thieme.de}

cME-Teilnahme

- Viel Erfolg bei lhrer CME-Teilnahme unter http://cme.thieme.de

- Diese Fortbildungseinheit ist 12 Monate online für eine CME-Teilnahme verfügbar.

- Sollten Sie Fragen zur Online-Teilnahme haben, unter

\section{CME-Fragen}

1 Welches Krankheitsbild wird nicht unter dem Begriff GERD subsumiert?

A erosive Refluxösophagitis verschiedener Schweregrade (ERD)

B nichterosive Refluxkrankheit (NERD)

C hypersensitiver Ösophagus

D axiale Hiatushernie

E Barrett-Ösophagus

Welche Aussage trifft zu? Ein Barrett-Ösophagus ...

A kann nicht histologisch diagnostiziert werden.

B entsteht bei über 50\% der Patienten mit diagnostizierter ERD.

C entwickelt sich am gastroösophagealen Übergang durch Umwandlung von Plattenepithel in spezialisiertes Zylinderepithel.

D wandelt sich in $12-13 \%$ pro Jahr in eine Neoplasie um.

E wird durch eine Impedanzmessung diagnostiziert.

3 Welche Aussage trifft zu? Die Prävalenz von GERD in der Schwangerschaft ...
A liegt deutlich unter $50 \%$ der schwangeren Frauen.
B nimmt mit Fortschreiten der Schwangerschaft zu.
C zeigt eine negative Korrelation mit dem Gestationsalter.
D zeigt eine negative Korrelation mit der Parität.
E liegt bei $90 \%$ der schwangeren Frauen.

\section{Wodurch wird GERD in der Schwangerschaft ausgelöst?}

A durch einen verminderten unteren Ösophagusspinktertonus, verursacht durch die schwangerschaftstypischen Hormone Progesteron und Östrogen

B durch einen erhöhten unteren Ösophagussphinkterdruck, verursacht allein durch den massiven intraabdominellen Druck

C durch die deutlich erhöhte Peristaltik des Ösophagus in der Schwangerschaft

D durch die genetische Prädisposition

E durch die zunehmende Darmkompression

$5 \quad$ Nach welchem Schema sollte GERD in der Schwangerschaft behandelt werden?
A Lebensstil-Änderungen - H2-RA - Antazida - Sucralfat - PPI
B Lebensstil-Änderungen - PPI - H2-RA - Antazida
C Lebensstil-Änderungen - Antazida - PPI -H2-RA
D Lebensstil-Änderungen Antazida - H2-RA - PPI
E PPI - H2-RA - Antazida - Lebensstil-Änderungen

\section{Was zählt nicht zu den in der Schwangerschaft empfohlenen Maßnahmen im Sinne der Lebensstil-Änderungen zur} Behandlung von GERD?
A mehrere kleinere Mahlzeiten über den Tag verteilt
B physiologische Gewichtszunahme in der Schwangerschaft
C Bier, Kaffee und Schokolade vermeiden
D Möglichst 3-4 Stunden vor dem Schlafengehen die letzte Mahlzeit einnehmen
E Zitrusfrüchte essen 


\section{Welche Aussage gilt nicht für Antazida?}

A Antazida sind Medikamente der ersten Wahl zu Behandlung von GERD in der Schwangerschaft.

B Magnesiumhaltige Antazida erhöhen das Risiko einer Eklampsie um 50\%.

C Antazida wirken neutralisierend auf die Magensäure.

D Die systemische Resorption von Antazida ist nur gering.

E Kalziumhaltige Antazida zeigen einen positiven Effekt auf die Vermeidung von Hypertonie und Präeklampsie.

\section{Welche Aussage zu H2-RA ist richtig?}

A Cimetidin ist der am häufigsten eingesetzte H2-RA bei GERD in der Schwangerschaft.

B Ranitidin ist Mittel der Wahl aus der Klasse der H2-RA zur Behandlung von GERD in der Schwangerschaft.

C Cimetidin zeigte in tierexperimentellen Untersuchungen eine androgene Wirkung.

D Famotidin ist der am besten untersuchte H2-RA zur Behandlung von GERD in der Schwangerschaft.

E Nizatidin zeigte als einziger H2-RA eine nachgewiesene Effektivität in der Behandlung von GERD in der Schwangerschaft.

9 Welche Aussage zur PPI-Behandlung in der Schwangerschaft trifft zu?

A PPI sind die wirkungsärmsten Medikamente zur Behandlung von GERD in der Schwangerschaft.

B PPI sollten nur bei persistierenden GERD-Symptomen trotz ausgeschöpfter medikamentöser Therapie zum Einsatz kommen

C PPI sind in der Schwangerschaft unbegrenzt einsetzbar.

D PPI sollten v. a. in der präkonzeptionellen Phase eingesetzt werden.

E Omeprazol ist bei Symptomen von GERD in der Schwangerschaft immer indiziert.

10 Welche Aussage trifft zu? Zur Behandlung von GERD in der Laktationsphase ...

A liegen wissenschaftlich gut validierte Untersuchungsergebnisse vor.

B sind PPI Mittel der ersten Wahl.

C sind Antazida Mittel der ersten Wahl.

D sollte entsprechend dem außerhalb der Schwangerschaft gültigen Step-down-Schema behandelt werden.

E besteht keine Gefahr, dass die eingesetzten Medikamente in die Muttermilch übertreten. 